
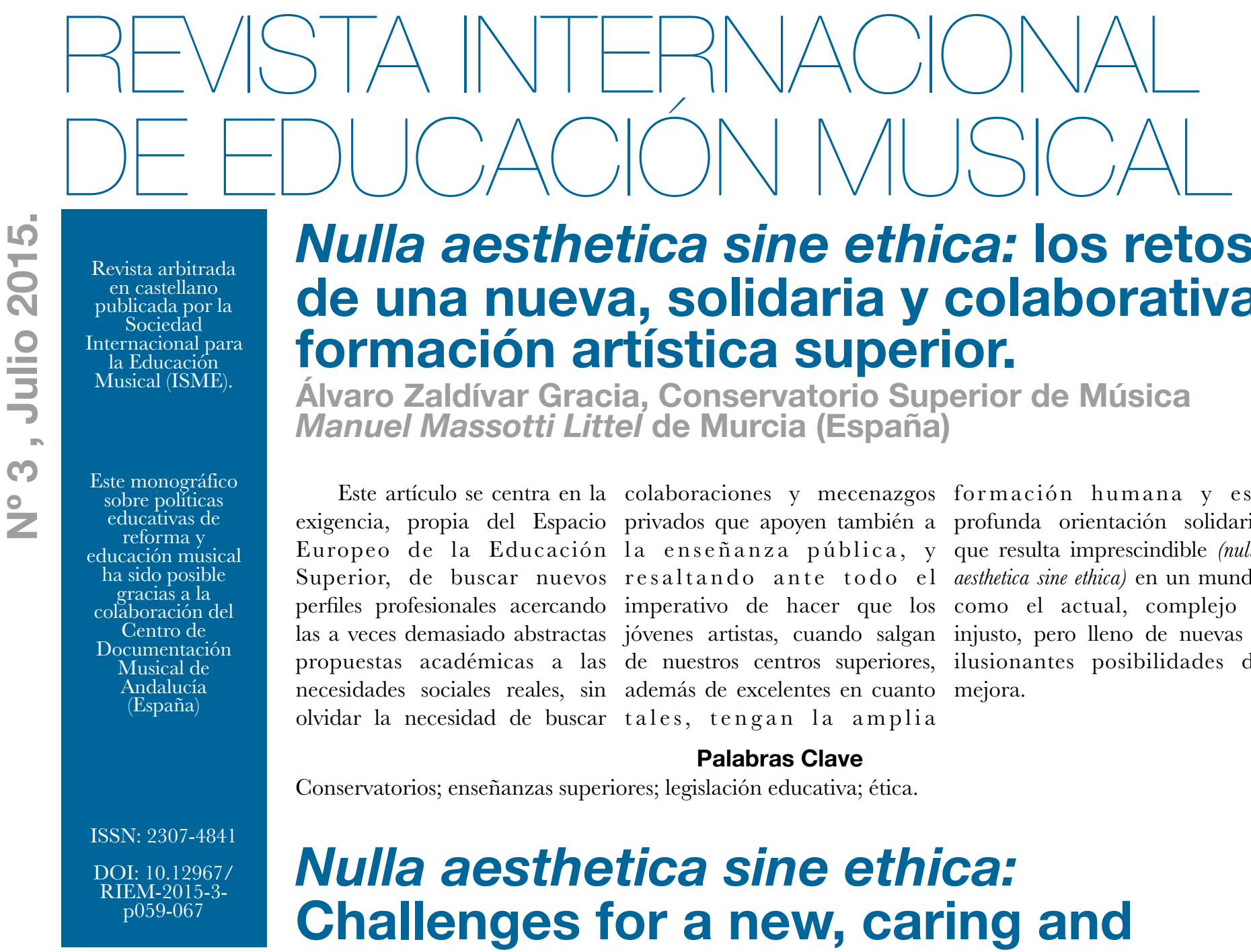

\title{
Nulla aesthetica sine ethica: los retos de una nueva, solidaria y colaborativa formación artística superior.
}

\author{
Álvaro Zaldívar Gracia, Conservatorio Superior de Música \\ Manuel Massotti Littel de Murcia (España)
}

Este artículo se centra en la colaboraciones y mecenazgos formación humana y esa exigencia, propia del Espacio privados que apoyen también a profunda orientación solidaria Europeo de la Educación la enseñanza pública, y que resulta imprescindible (nulla Superior, de buscar nuevos resaltando ante todo el aesthetica sine ethica) en un mundo perfiles profesionales acercando imperativo de hacer que los como el actual, complejo e las a veces demasiado abstractas jóvenes artistas, cuando salgan injusto, pero lleno de nuevas e propuestas académicas a las de nuestros centros superiores, ilusionantes posibilidades de necesidades sociales reales, sin además de excelentes en cuanto mejora.

olvidar la necesidad de buscar tales, tengan la amplia

\section{Palabras Clave}

Conservatorios; enseñanzas superiores; legislación educativa; ética.

\section{Nulla aesthetica sine ethica: Challenges for a new, caring and collaborative higher artistic training \\ Álvaro Zaldívar Gracia, Manuel Massotti Littel Murcia Higher Conservatory (Spain)}

This paper copes with the collaborations and patronages to this current world, so complex Higher Education European support public teaching. It also and unjust but, likewise, full of Space demand of looking for stresses out the imperative for new and exciting possibilities for new professional profiles in order the graduate young artists to be improvement.

to get together the so often no only excellents, but also a abstracts academic proposal with human training with a deep real social needs. It also discusses solidarity approach, a driving the need of searching for private need (nulla aesthetica sine ethica) in

\section{Keywords}

Conservatoires; higher teaching; educational law; ethics.

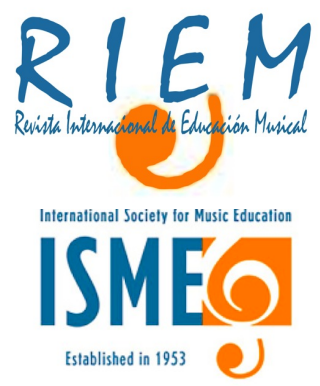




\title{
Nulla aesthetica sine ethica: los retos de una nueva, solidaria y colaborativa formación artística superior.
}

\author{
por Álvaro Zaldívar Gracia, Conservatorio Superior de Música Manuel Massotti Littel de Murcia (España).
}

Dejando al margen, por razones de oportunidad y tiempo, todo el rico debate que puede derivarse del espacio y forma que ha de ocupar - con asignatura propia o sin ella- la ética en la enseñanza general (y permítasenos que no entremos aquí en un debate sobre el posible alcance y la orientación de la misma, ni mucho menos su discriminación con la moral, que dejamos a los correspondientes expertos), de tan sutiles matices filosóficos-pedagógicos como enconadas posturas político-ideológicas, en este artículo sólo se pretende, humildemente, iniciar la cuestión de la, no sólo posible sino más bien obligada, competencia ética dentro de las Enseñanzas Artísticas Superiores. Esas Enseñanzas, dirigidas a la formación de los más avanzados profesionales de la música, la danza, el arte dramático, la conservación y restauración de bienes culturales, determinadas artes plásticas - cerámica y vidrio- y el diseño que, en España, entre la Ley Orgánica de Ordenación General del Sistema Educativo -LOGSE- de 1990 y la Ley Orgánica de Educación -LOEde 2006 y con la Ley Orgánica para la Mejora de la Calidad Educativa -LOMCE- de 2013, no sólo fueron creadas -hace muchos años-, sino que hoy permanecen organizadas fuera de la universidad, aunque hayan sido progresivamente incardinadas en algunos de los diversos parámetros de la Educación Superior: primero con la general equiparación de sus titulaciones a las universitarias, en 1990, y luego, a partir de 2006, con la incorporación de la estructura y organización de las enseñanzas al Espacio Europeo de Educación Superior, a través del sistema de transferencia de créditos, la separación de niveles entre grado y posgrado, etc.

En definitiva, y con toda la sencillez de una propuesta inicial, si hace casi ya una década intenté aportar mi pequeño grano de arena en la difusión del debate científico/ metodológico sobre una legítima investigación «desde la práctica artística» en las enseñanzas superiores no universitarias (Zaldívar, 2005, 2006, 2008ª 2008 ${ }^{\text {b }}$ 2010, 2011, 2013), ahora también muy modestamente intento que se introduzca también en nuestras aulas y pasillos, en los despachos y salas de juntas de nuestros centros y, quizás, también en los de las administraciones educativas, un debate más filosófico/pedagógico sobre la formación ética en los conservatorios y demás centros superiores de Enseñanzas Artísticas, tema poco tratado en nuestro sector que, sin embargo, tuve la suerte de experimentar como pionero participante, en el curso 2011-12, dentro del Proyecto de Innovación que, desde el Centro de Profesores y Recursos (I) de Murcia, y coordinado por mi admirado colega y amigo Antonio Narejos, se centró entonces en La programación de las actividades de Acción Solidaria en los Estudios Superiores de Música de la Región de Murcia, como una primera aproximación concreta en tales tareas.

Debemos, sin embargo, antes de continuar con lo relativo a la formación ética como tema aún pendiente en nuestras Enseñanzas Artísticas Superiores, detenernos brevemente esbozando apenas dos marcos previos: el campo donde esta propuesta se inserta, es decir, en los perfiles formativos de tales enseñanzas; y la presencia (e influencia, en su caso) de un imprescindible nuevo mecenazgo que haga posibles nuevos proyectos, tanto para la enseñanza pública como para la privada, más allá de lo sostenible desde la mejor administración de los escasos recursos públicos.

\section{Nuevos perfiles profesionales: propuestas académicas y necesidades sociales.}

Insistamos, en primer lugar, recordando que, en contra del modelo vigente del sistema universitario español, las enseñanzas artísticas superiores establecidas en la LOE dentro del nivel de Grado (para evitar problemas con las reiteradas sentencias del Tribunal Supremo que anulan el término para las titulaciones pero no así para el nivel de cualificación, de esta manera es denominado, con independencia de su condición intra o extrauniversitaria, en el Real Decreto 1027/2011, de 15 de julio, por el que se establece el Marco Español de Cualificaciones para la Educación Superior -MECES-), modificado por el Real Decreto 96/2014 (Ministerio de Educación, Cultura y Deporte, 2014) son estudios que vienen ya prediseñados en sus títulos, especialidades, competencias, materias fundamentales y respectivos perfiles profesionales por normas básicas estatales que los enumeran y desarrollan inicialmente, a partir de lo señalado por la Ley estatal. Esta norma educativa, del año 2006, no ha cambiado sustancialmente (aunque ha mejorado, sobre todo por la inserción en el Espacio Europeo de la Educación Superior y lo que ello conlleva) el marco ya otorgado por la LOGSE en 1990: es decir, hace 23 años, aunque obviamente entre una y otra norma se han dado muy fuertes transformaciones sociales, económicas y culturales. El nivel de máster (según el mismo Real Decreto del MECES, si bien las referidas Sentencias del Tribunal Supremo rechazaron los recursos universitarios sobre su apropiada y mantenida denominación específica como máster en Enseñanzas Artísticas, que obviamente en nada puede confundirse con los del título de máster universitario), es un terreno de formación de posgrado artístico que abre la LOE, y que ahora está comenzando su desarrollo efectivo, a partir de la aplicación del Real Decreto 1614/2009 - parcialmente modificado por el Real Decreto 21/2015 (Ministerio de Educación Cultura y Deporte, 2015) - donde, siguiendo más de cerca el modelo universitario, no se predetermina con normas estatales sus posibles especialidades o materias, que quedan en manos de las propuestas presentadas por parte de las Comunidades Autónomas y de la correspondiente recepción de las agencias evaluadoras (primero la ANECA, estatal, a petición de Valencia; la ACSUCYL para su propio territorio - Castilla y León-, y luego la ACAP a instancia de la Comunidad de Madrid). Con esta nueva libertad, que permite mayor competitividad legítima, exigiendo mucha más autoexigencia y mejor programación previa que el simple desarrollo autonómico de unos títulos superiores de nivel de grado tan preconfigurados, estos nuevos másteres en Enseñanzas 
Artísticas, que han mostrado ya el curso 2013/2014 los primeros títulos oficiales expedidos, sin duda ofrecen poco a poco un abanico mucho más abierto de novedosos perfiles profesionales que pueden con libertad adaptarse a las cambiantes necesidades sociales.

Como hasta el momento no ha sido aún aprobada la norma estatal que dicte las preceptivas directrices propias de una profesión regulada (para el correspondiente máster de formación de profesorado de Enseñanzas Artísticas profesionales), los actuales másteres artísticos en marcha se orientan, lógicamente, con plena libertad, hacia una formación avanzada que mira tanto al perfeccionamiento instrumental o creativo práctico como al inicio de la tarea investigadora, salvo perfiles más singulares, como la reciente propuesta de la Comunidad de Madrid de crear un máster de música y artes escénicas (teatro y danza) en y para la discapacidad, con el que se quieren hacer presentes unos colectivos normalmente ausentes -salvo excepciones más o menos tradicionales, como la tan veterana y prestigiosa de tantos ilustres músicos invidentes- en el terreno de las Enseñanzas Artísticas Superiores. Un esperanzador nivel de posgrado, como bien se ve, que más que dejar sólo un espacio a la cuestión ética (que está también obligadamente presente en el nivel de Grado, según veremos más adelante), potencia aún más la exigencia de abrir una profunda reflexión humanística - no sólo artística- sobre las formas y contenidos de una formación que vaya más allá de los aspectos técnicos de los futuros profesionales de las artes, en general, y de la música en particular.

\section{Colaboraciones y mecenazgos en la oferta pública}

Aunque no conozco estudios solventes que nos permitan afirmarlo probadamente, parece difícil negar que la sociedad española actual ha perdido ese mayoritario sentimiento de exigencia y necesidad de formación y disfrute artístico que tan felizmente se expandió en la transición democrática de los años setenta y ochenta (multiplicando en poco tiempo los conservatorios y escuelas, los auditorios y ciclos de conciertos). Ese afán compartido hizo que hasta hace muy poco la oferta pública de las Enseñanzas Artísticas se entendiese aún como una obligación -dentro de las enseñanzas no obligatorias- de las administraciones educativas (e incluso, en cierto sentido y para determinados ámbitos, también de administraciones no propiamente educativas, como los ayuntamientos). Sin embargo, la llamada crisis económico-financiera de los últimos años, y la consiguiente aceptación mayoritaria de un nuevo modelo de orden y gasto social recortado, presentado como obligado e inapelable, parece haber hecho olvidar que la formación artística sigue siendo un derecho, una actividad esencial cuyo valor vertebrador, que tuvo mucho que ver con el papel de los artistas en el desarrollo de las libertades democráticas en la España del último cuarto del siglo $\mathrm{XX}$, sigue vigente y es quizás hoy, incluso, más necesario que nunca.

Desde una perspectiva humana, desde luego, pero incluso también siguiendo sólo imperativos económicos, el gasto educativo en general, y creativo/artístico en especial, es una inversión inmejorable en una economía globalizada que valora particularmente esos llamados intangibles, y más aún, una inversión del todo necesaria para aquellas naciones, como la nuestra, que deben competir con creatividad e inteligencia frente a otros estados ricos en preciadas materias primas o en potentes y costosas industrias. En suma, la sociedad española se ha transformado, y el papel de "dinamizador popular» de las artes -marcadas por el creciente mercantilismo y la desideologización del inicio del nuevo milenio- se ha ido desdibujando, con la correspondiente reducción de esa «necesidad social» de la formación artística que caracterizó la España del fin del novecientos, con sus conservatorios llenos de alumnos, los conciertos - clásicos y populares- repletos de público y un asombroso crecimiento institucional de todo tipo de infraestructuras culturales (auditorios, orquestas estables, etc.).

Obviamente, en esta nueva situación, con las arcas de las instituciones públicas marcadas por la reducción drástica de ingresos y con una dolorosa reducción también de gastos entre los que ya casi todo puede entenderse incluido y por tanto estar en riesgo cierto (y no sólo un cierto riesgo) de eliminación asumida de modo conformista, la oferta pública educativo-cultural en general, y artística en particular, necesita urgentemente buscar nuevas ayudas e ingresos que puedan coadyuvar a mantenerse. Una búsqueda de mecenazgo y patrocinio (que a veces parece lo mismo pero que no lo es, aunque pueda sumarse favorablemente si se hace bien), que acaba de comenzar pero que no sabemos cómo se estimulará tributariamente, ni por cuánto tiempo deberá durar ni en qué puntos crecerá en protagonismo cualitativo o cuantitativo hasta resultar, quizás, más fundamental que auxiliar en determinadas circunstancias. Sin olvidar que ese patrocinio puede, más que en el caso del mecenazgo aunque tampoco quede éste del todo exento, conllevar ciertos condicionamientos: es decir, que tales ayudas no salen gratis, buscando a veces compensaciones que pueden ser soportables y hasta estimulantes, pero en otros casos priorizando criterios e incluso imponiendo vetos inaceptables para una legítima iniciativa pública. He aquí otro de los retos esenciales de un futuro donde, entre las necesidades vitales, y como feliz paradoja entre tanto debate economicista, se extiende también la exigencia de una formación ética como base de un comportamiento ejemplar que tanto más se requiere cuanto más se perciben los terribles efectos, individuales y sociales, de su a veces palmaria ausencia.

\section{Ética en la formación universitaria y en la formación artística superior: ámbito estatal.}

$\mathrm{Al}$ regresar al tema central de nuestra propuesta, en este mundo de viejos esquemas educativos que han de hacer frente a nuevas necesidades sociales, en una sociedad que siente y valora el arte hoy de modo bien distinto a hace unas pocas décadas, y donde iniciativas públicas y privadas están obligadas a entenderse, surge una pregunta derivada de esta intersección de tantas circunstancias socioeducativas que, al concretarse en la formación artística, se introduce en el complejo binomio ética/estética. Podríamos preguntarnos, por tanto, por el espacio que le deja a la estética la búsqueda de una ética acorde con estos complejos tiempos... pero en esta ocasión, por razones obvias, debemos cuestionarnos acerca de justo su complementario: es decir, qué papel tiene la ética en una formación tan claramente estética (aunque más bien reducida ésta a su más concreto «hacer» técnico del 
creador o intérprete) como es la propia de las Enseñanzas Artísticas Superiores, sean universitarias o no. Aunque, como resulta asimismo lógico, no vamos a centrarnos en lo universitario, sí que partiremos de ello ${ }^{1}$ para entrar en lo que aquí nos atañe para esta convocatoria, que son las Enseñanzas Artísticas Superiores organizadas actualmente al margen de la universidad.

Insertado dentro de la formación universitaria (y en su ámbito estatal) destaca el Real Decreto 861/2010, de 2 de julio, por el que se modifica el Real Decreto 1393/2007, de 29 de octubre, donde se establece la ordenación de las enseñanzas universitarias oficiales (Boletín Oficial del Estado de 3 de julio de 2010), en cuyo Anexo I, Memoria para la solicitud de verificación de títulos oficiales, se señala que "el proyecto constituye el compromiso de la institución sobre las características del título y las condiciones en las que se van a desarrollar las enseñanzas" (p. 54.864). En el apartado 3, Competencias, de esta misma norma, se señalan las "competencias generales y específicas que los estudiantes deben adquirir durante sus estudios, y que sean exigibles para otorgar el título. Las competencias propuestas deben ser evaluables. Deberán tenerse en cuenta los principios recogidos en el artículo 3.5 de este Real Decreto" (ibíd.).

Vayamos, pues, al citado Real Decreto 1393/2007, de 29 de octubre, por el que se establece la ordenación de las enseñanzas universitarias oficiales (Boletín Oficial del Estado de 30 de octubre de 2007), en cuyo Artículo 3. Enseñanzas universitarias y expedición de títulos, se dispone:

Entre los principios generales que deberán inspirar el diseño de los nuevos títulos, los planes de estudios deberán tener en cuenta que cualquier actividad profesional debe realizarse:

a) Desde el respeto a los derechos fundamentales y de igualdad entre hombres y mujeres, debiendo incluirse, en los planes de estudios en que proceda, enseñanzas relacionadas con dichos derechos.

b) Desde el respeto y promoción de los Derechos Humanos y los principios de accesibilidad universal y diseño para todos de conformidad con lo dispuesto en la disposición final décima de la Ley 51/2003, de 2 de diciembre, de Igualdad de oportunidades, no discriminación y accesibilidad universal de las personas con discapacidad, debiendo incluirse, en los planes de estudios en que proceda, enseñanzas relacionadas con dichos derechos y principios.

c) De acuerdo con los valores propios de una cultura de paz y de valores democráticos, y debiendo incluirse, en los planes de estudios en que proceda, enseñanzas relacionadas con dichos valores (pp. 44.038 y 44.039).

Acudiendo de nuevo a la Memoria de Verificación (puntos 3.2 y 3.3 del este Real Decreto), entre las competencias básicas que se garantizarán como mínimo, entre otras (como "que los estudiantes sepan aplicar sus conocimientos a su trabajo o vocación de una forma profesional y posean las competencias que suelen demostrarse por medio de la elaboración y defensa de argumentos y la resolución de problemas dentro de su área de estudio" (p. 44.046), o bien "que los estudiantes puedan transmitir información, ideas, problemas y soluciones a un público tanto especializado como no especializado" (ibíd.), etc.), destacamos aquí las dos siguientes, dentro del nivel de Grado y de Máster - no así en el caso del doctorado-, respectivamente:

Que los estudiantes tengan la capacidad de reunir e interpretar datos relevantes (normalmente dentro de su área de estudio) para emitir juicios que incluyan una reflexión sobre temas relevantes de índole social, científica o ética.

Que los estudiantes sean capaces de integrar conocimientos y enfrentarse a la complejidad de formular juicios a partir de una información que, siendo incompleta o limitada, incluya reflexiones sobre las responsabilidades sociales y éticas vinculadas a la aplicación de sus conocimientos y juicios (ibíd., p. 44.046).

No es sino una copia directa de lo arriba referido lo que se señala para la formación artística superior (en su ámbito estatal), como se manifiesta en el Real Decreto 1614/2009, de 26 de octubre, por el que se establece la ordenación de las enseñanzas artísticas superiores reguladas por la Ley Orgánica 2/2006, de 3 de mayo, de Educación (Boletín Oficial del Estado, de 27 de octubre de 2009), en cuyo artículo 3. Enseñanzas artísticas superiores y expedición de títulos, se recuerda nuevamente que:

4. Entre los principios generales que deberán inspirar el diseño de los nuevos títulos, los planes de estudios deberán tener en cuenta que cualquier actividad profesional debe realizarse:

a) Desde el respeto a los derechos fundamentales y de igualdad entre hombres y mujeres, debiendo incluirse, en los planes de estudios en que proceda, enseñanzas relacionadas con dichos derechos.

b) Desde el respeto y promoción de los Derechos Humanos y los principios de accesibilidad universal y diseño para todos de conformidad con lo dispuesto en la disposición final décima de la Ley 51/2003, de 2 de diciembre, de Igualdad de oportunidades, no discriminación y accesibilidad universal de las personas con discapacidad, debiendo incluirse, en los planes de estudios en que proceda, enseñanzas relacionadas con dichos derechos y principios.

c) De acuerdo con los valores propios de una cultura de paz y de valores democráticos, y debiendo incluirse, en los planes de estudios en que proceda, enseñanzas relacionadas con dichos valores (p. 89.745).

Relativo ya en concreto para música, pues en este campo se centra la presente convocatoria, y aún en el ámbito estatal, el Real Decreto 631/2010, de 14 de mayo, por el que se regula el contenido básico de las enseñanzas artísticas superiores de Grado en Música establecidas en la Ley Orgánica 2/2006, de 3 de mayo, de Educación (Boletín Oficial del Estado de 5 de junio de 2010), señala reiteradamente en el final del preámbulo que "entre los principios generales que deberán inspirar el diseño de los nuevos títulos, los planes de estudios tendrán en cuenta que cualquier actividad profesional debe realizarse desde el respeto a los derechos fundamentales y de igualdad entre hombres y mujeres, desde la promoción de los Derechos Humanos y con los valores propios de una cultura democrática" (p. 48.481). Y en su artículo 6.2, Contenido básico de los planes de estudios conducentes a la obtención del Título de Graduado o Graduada en Música, se dice que "los planes de estudios conducentes a la obtención del Título de Graduado o Graduada en Música, deberán contener las competencias transversales, las competencias generales, y las competencias específicas y los perfiles profesionales definidos para cada una de las especialidades, que se determinan en el Anexo I del presente Real Decreto" (p. 48.483). Es en el referido Anexo I donde podemos leer, entre las Competencias transversales del Graduado o Graduada en Música, que "al finalizar sus estudios los Graduados y Graduadas en Música deben poseer”, entre otras (por ejemplo, "Organizar y planificar el trabajo de 
forma eficiente y motivadora", o bien "Comprender y utilizar, al menos, una lengua extranjera en el ámbito de su desarrollo profesional", etc.), la siguiente: "Desarrollar en la práctica laboral una ética profesional basada en la apreciación y sensibilidad estética, medioambiental y hacia la diversidad" (p. 48.488).

\section{Desarrollo autonómico en el ámbito musical}

Otra cosa sucede en el desarrollo autonómico, centrándonos ya sólo en las Enseñanzas Artísticas Superiores de música. Por ejemplo, en el caso de la Comunidad de Madrid, en cuyo Decreto 36/2010, de 2 de junio, del Consejo de Gobierno, por el que se establece el Plan de Estudios para la Comunidad de Madrid, de las enseñanzas artísticas superiores de Grado en Música (BOCM de 16 de junio de 2011), no figuran separada y ordenadamente las competencias transversales, generales y específicas, sino que directamente se incorporan en las diversas asignaturas, centradas normalmente en los aspectos técnicos más concretos de las mismas y con sólo esporádicas referencias a las transversales (como cuando se incluye la competencia de «organizar y planificar el trabajo de forma eficiente y motivadora» dentro de las asignaturas tan distintas como Historia de la Música, Análisis o Estética y Filosofía de la Música).

De Andalucía podemos destacar el Decreto 260/2011, de 26 de julio, por el que se establecen las enseñanzas artísticas superiores de Grado en Música en Andalucía (BOJA de 23 de agosto de 2011) en cuyo Artículo 14.2 Evaluación y convocatorias se dispone que "los criterios de evaluación correspondientes al plan de estudios son los que se determinan en el Anexo III. Los centros docentes especificarán los criterios de evaluación para cada asignatura" (p. 40). Y en el citado Anexo III, Criterios de evaluación, dentro de los transversales, entre otros, "demostrar capacidad para recoger y analizar y sintetizar información significativa y gestionarla de forma adecuada" (p. 90), o bien, "demostrar dominio de la metodología de la investigación en la generación de proyectos, ideas y soluciones viables" (ibíd.), etc., se incluye: "Demostrar la aplicación de principios éticos en el desarrollo profesional" (ibíd.).

En el previo Anexo II, Contenidos y competencias, donde figuran las asignaturas con la atribución concreta de todas las competencias (enumeradas ordenadamente al final del mismo), podemos ver que la última citada corresponde a la competencia transversal del graduado o graduada en música número 11 («desarrollar en la práctica laboral una ética profesional basada en la apreciación y sensibilidad estética, medioambiental y hacia la diversidad»), y más que curiosamente ese número aparece como competencia a alcanzar tanto en Historia de la Música o en Sociología y Estética de la música, como en Lenguaje y teoría musical y Análisis, o también en ¡Práctica armónica en instrumentos polifónicos!, por citar sólo las primeras asignaturas del Anexo.

Recurriendo a continuación a Valencia en su Decreto 48/2011, de 6 de mayo, del Consell, por el que se establece la ordenación de las enseñanzas artísticas superiores y se determina el marco normativo para la implantación de los planes de estudios correspondientes a los títulos oficiales de graduado o graduada en las diferentes enseñanzas artísticas superiores, en el ámbito de la Comunitat Valenciana (DOCV de
10 de mayo de 2011), y en su desarrollo la Orden 24/2011, de 2 de noviembre, de la Conselleria de Educación, Formación y Empleo, por la que se establecen y autorizan los planes de estudio de los centros de enseñanzas artísticas superiores de música dependientes del ISEACV, conducentes a la obtención del título de Graduado o Graduada en Música. (DOCV de 10 de noviembre de 2011), dentro de su Anexo I, dedicado a las Competencias transversales del título de Graduado o Graduada en Música, entre otras (por ejemplo, "solucionar problemas y tomar decisiones que respondan a los objetivos del trabajo que se realiza", o bien "buscar la excelencia y la calidad en su actividad profesional" - $p$. 36.597-, etc.), se incluye, como ya se ha visto en el caso andaluz: "CT 11. Desarrollar en la práctica laboral una ética profesional basada en la apreciación y sensibilidad estética, medioambiental y hacia la diversidad". Pero además, dentro de las Competencias específicas del Título de Graduado o Graduada en Música en la especialidad de Pedagogía podemos leer, entre otras (caso de "conocer los elementos constitutivos de la música mostrando un alto dominio en percepción auditiva, lectura, análisis, escritura, improvisación y creación musical, y ser capaz de interrelacionar todo ello para aplicarlo y utilizarlo adecuadamente en el desarrollo de la propia actividad" (p. 36.601), o también "conocer los fundamentos de acústica musical, organología y sus aplicaciones en la práctica musical" -p. 36.602-, etc.), las siguientes de clara orientación ética:

CE 5 Ser capaz de desarrollar una práctica educativamusical, como artista y formador musical orientada a la comunidad.

CE 6 Contextualizar la pedagogía musical en el tiempo actual y en los diferentes ámbitos culturales, reflexionando de forma crítica sobre la función y resultados que la práctica de la educación musical puede aportar a la mejora de la persona y de la sociedad (pp. 36601 y 36602).

Otra cosa es comprobar más adelante, en el Anexo II de la misma norma, que la citada competencia transversal (CT 11) aparece en asignaturas en las que, como antes vimos, parece complicado encajar, caso de Composición o Instrumentación y Orquestación, por ejemplo, y más todavía en ¡Piano aplicado!. Y las arriba citadas competencias específicas de la especialidad de pedagogía tampoco se salvan de su problemática adscripción a algunas asignaturas, pues la referida CE 5 figura en, entre otras, Historia de la Música o Estética, pero además en Análisis y en Orquesta o Música de Cámara, y también en ¡Práctica armónico-contrapuntística! Mientras que la CE 6 aparece en Gestión musical pero, asimismo, en el Taller específico de la materia Formación Instrumental complementaria... Y figurando ambas (CE5 y CE 6) lógicamente en Psicopedagogía, Didáctica general de la música y Didáctica específica, etc., también así sucede en ¡Instrumento principal!.

Merecería la pena revisar la adscripción de no pocas competencias en aquellas normativas que se han tomado la molestia de relacionarlas con las distintas asignaturas y cursos: por ejemplo, en la Orden valenciana antes citada, la Competencia Transversal número 5, "Comprender y utilizar, al menos, una lengua extranjera en el ámbito de su desarrollo profesional”, quizás no debería estar sólo en asignaturas como Idioma aplicado y las correspondientes lenguas que poseen su Fonética aplicada, así como en Organología o Trabajo de Fin de Grado pues, cpor qué no aparece también en Historia de la Música, en Metodologías de la investigación 
o en Gestión Musical, donde los idiomas no son menos relevantes?

Insistiendo en que esta tan apresurada y recortada revisión, por la que ruego anticipadas disculpas y pido la comprensión general ante una selección inicial meramente ilustrativa, no es cosa de un lugar o normativa concretas, sino más bien una situación general, en el antes referido desarrollo andaluz, la competencia transversal que acabamos de comentar, que mantiene la numeración (CT5), aparece obviamente en Fonética e idiomas aplicados al canto, y lo hace también en Concertación como en Análisis y en Introducción a la gestión cultural y otras afines, apareciendo asimismo en Composición y en Instrumentación. Y aunque no figura en Organología y acústica, está en algunos de los Trabajos de Fin de Grado: ausente de los de Composición o Dirección, sí figura en el de Flamenco, especialidad que contiene la CT 5 en... Armonía Aplicada a la Guitarra Flamenca, en Música de Cámara Flamenca y, puede que con mucha más lógica, en Sociología del Flamenco.

\section{Algunas propuestas para el futuro}

Llena de paradojas está, por tanto, la situación. En esta primera aproximación, que es obligadamente mucho más que incompleta (aunque puede que sirva como invitación para posibles futuras investigaciones solventes a los interesados en el tema), comprobamos que la competencia ética está presente en algunos casos, si bien su adscripción a determinadas asignaturas, lógica y coherente, contrasta en otros con relaciones bastante más discutibles. Y habría que entrar, ya en detalle, para proponer lo que debiera más en concreto suponer, saber cómo se puede eficazmente alcanzar y evaluar adecuadamente dicha competencia en cada una de esas asignaturas, así como hacer un seguimiento a fondo de su acogida en el aula y, en su caso, la correspondiente efectividad en el desarrollo profesional.

Aunque estas apretadas reflexiones terminan forzosamente aquí, esperamos que sí puedan, al menos, llegados al final del tiempo previsto, comenzar un fructífero diálogo, y la pregunta sigue ahora más vigente que al iniciar el artículo: ¿dónde está la competencia ética, de verdad, en nuestros currículos, en nuestros centros, en nuestras aulas?, ¿dónde y cómo podría o debería ser? Pudiendo avanzar incluso en muchas otras orientaciones derivadas, pues deberíamos también determinar quizás qué puede tener que ver esta propuesta formativa en nuestros centros con las -más necesarias que meramente posibles- acciones de organizaciones solidarias que desde el arte y la música llevan a cabo tareas parejas a las de otros generosos colectivos profesionales (caso de, por ejemplo, Músicos solidarios sin fronteras $^{2}$, actualmente con sedes en Vitoria y Valencia). O incluso, yendo mucho más allá, ya directamente en el ámbito profesional, plantear la relación con un efectivo código deontológico que, al estilo de los tan celebrados y populares propios de médicos o periodistas, se pudiera imponer voluntariamente entre los artistas en general (hay una reciente propuesta española de código deontológico del Instituto de Arte Contemporáneo ${ }^{3}$, aprobada en la Asamblea General de estos profesionales dedicados al arte actual, celebrada en mayo de 2011, y que cita como precedente el código del Consejo de Críticos de Artes Visuales) y los músicos en particular, no menos faltos de estándares éticos profesionales a pesar de esa tan presunta como ingenua incontaminación del arte sonoro. Es más que curioso que, como colectivo, y salvo las ya sabidas imposiciones gubernamentales totalitarias, o muy singulares casos públicamente comprometidos, los músicos cultos normalmente pretendan estar casi siempre al margen de todo juicio ético e incluso por encima de toda ideología (lo que permite a la aislada historiografía musical usar -en contra de lo que sucede en la mayor parte de las demás artes- la etiqueta de «nacionalista» para quienes a veces sólo han practicado ciertos desideologizados folklorismos en algunas de sus obras más costumbristas, al tiempo que cumplían a rajatabla ese famoso consejo de «mejor no meterse en política»).

No nos desanimemos si terminamos con más preguntas y sin respuestas. Este artículo, como ya se dijo en un principio, sólo quiere abrir un debate que se centra en la exigencia, propia del Espacio Europeo de la Educación Superior, de buscar nuevos perfiles profesionales acercando las a veces demasiado abstractas propuestas académicas a las necesidades sociales reales (sin olvidar aquí la doble necesidad de buscar colaboraciones y mecenazgos privados que apoyen también a la enseñanza pública) y resaltando ante todo el imperativo de hacer que los jóvenes artistas, cuando salgan de nuestros centros superiores, además de excelentes en cuanto tales, tengan la amplia formación humana y esa profunda orientación solidaria que resulta imprescindible (nulla aesthetica sine ethica) en un mundo como el actual, complejo e injusto, pero lleno de nuevas e ilusionantes posibilidades de mejora. Lo que no es poco, precisamente.

\section{Notas}

${ }^{1}$ No es éste el lugar donde hacer un cumplido estado de la cuestión del debate sobre la formación ética en el ámbito universitario, pero es obvio que lo ya desarrollado en este campo es mucho más que un precedente para nuestra propuesta. $\mathrm{Al}$ menos recordemos aquí dos importantes aportaciones publicadas en Teoría de la Educación. Revista interuniversitaria de Teoría de la Educación, de las ediciones de la Universidad de Salamanca: la realizada por los profesores catalanes Francisco Esteban Bara y María Rosa Buxarrais Estrada titulada «El aprendizaje ético y la formación universitaria: más allá de la casualidad» (2004), o la de los profesores de la Facultad de Filosofía y Ciencias de la Educación de la Universidad de Valencia, Rafaela García López, Auxiliadora Sales Ciges, Odet Moliner García y Reina Ferrández Barueco, titulada «La formación ética profcesional desde la perspectiva del profesorado universitario» (2009). Los dos primeros profesores citados, con la firma inicial de Miquel Martínez Martín, ya habían publicado antes otra interesante contribución titulada «La universidad como espacio de aprendizaje ético» (2002). Para que no se vea esta cuestión limitada sólo a los ámbitos de los profesionales de la ciencia educativa, recordemos al menos que dos profesores del departamento de proyectos de ingeniería, de la Universidad Politécnica de Valencia, Alejandra Boni Aristizabal y José Félix Lozano Aguilar, realizaron hace ya tiempo una interesante comunicación titulada «El aprendizaje ético en la universidad y su relación con las competencias transversales en el diseño de los nuevos planes de estudios», dentro del III Congreso Internacional Docencia Universitaria e Innovación desarrollado en Gerona entre el 30 de junio y el 2 de julio de 2004. Y señalemos ya finalmente, para que se vea que se trata de un tema aún vivo y actual, cómo el antes citado Francisco Esteban Bara, con la 
firma previa de Begoña Román Maestre, filósofa y pedagogo, respectivamente, ambos de la universidad de Barcelona, son los autores de una relevante comunicación presentada al Congreso Internacional Docencia Universitaria e Innovación, La universidad: una institución de la sociedad, celebrado en la Universidad Pompeu Fabra de Barcelona, entre los días 4 y 6 de julio de 2012, titulada sugestivamente «Aporías de la formación ética en la Universidad de hoy».

${ }^{2}$ http://www.musicossinfronteras.es

${ }^{3}$ http://www.iac.org.es/wp-content/uploads/2011/05/C $\%$ C3 \%B3digo-deontol $\%$ C3\%B3gico-del-IAC.pdf

\section{Referencias citadas}

Bara, F. E. y Buxarrais, M. R. (2004). El aprendizaje ético y la formación universitaria: más allá de la casualidad. Teoría de la Educación, 16, 91-108.

Bara, F. E., Buxarrais, M. R. y Martínez, M. (2002). La universidad como espacio de aprendizaje ético. Revista Iberoamericana de educación, 29, 17-44. Disponible en http:// www.rieoei.org/rie29a01.htm.

Boni, A. y Lozano, J. F. (2004). El aprendizaje ético en la universidad y su relación con las competencias transversales en el diseño de los nuevos planes de estudio. Comunicación presentada dentro del III Congreso Internacional Docencia Universitaria e Innovación. Gerona, del 30 de junio al 2 de julio.

Consejería de Educación de la Comunidad de Madrid (2010). Decreto 36/2010, de 2 de junio, del Consejo de Gobierno, por el que se establece el Plan de Estudios para la Comunidad de Madrid, de las enseñanzas artísticas superiores de Grado en Música. Boletín Oficial de la Comunidad de Madrid, 141, 11-253. Disponible en http://www.emes.es/ Link C lick.as px? fil et i cket = ow 4 w Z i q y Kv I $\% 3 \mathrm{D} \&$ tabid $=46 \& \mathrm{mid}=355 \&$ forcedownload $=$ true $\&$ name $=\mathrm{D}$ _36_02061606-2010_grado_musica.pdf.

Consejería de Educación de la Comunidad Valenciana (2011). Decreto 48/2011, de 6 de mayo, del Consell, por el que se establece la ordenación de las enseñanzas artísticas superiores y se determina el marco normativo para la implantación de los planes de estudios correspondientes a los títulos oficiales de graduado o graduada en las diferentes enseñanzas artísticas superiores, en el ámbito de la Comunitat Valenciana. Boletín Oficial de la Comunidad Valenciana, 6517, 18.092-18.102. Disponible en http:// www.iseacv.es $/$ fichero.php?file=normativa/decreto-48-2011ordenaci-n-eeaass-cv_52.pdf.

Consejería de Educación, Formación y Empleo de la Comunidad Valenciana (2011). Orden 24/2011, de 2 de noviembre, de la Conselleria de Educación, Formación y Empleo, por la que se establecen y autorizan los planes de estudio de los centros de enseñanzas artísticas superiores de música dependientes del ISEACV, conducentes a la obtención del título de Graduado o Graduada en Música. Boletin Oficial de la Comunidad Valenciana, 6648, 36.595-36.801. Disponible en http://www.docv.gva.es/datos/2011/11/10/ pdf/2011_11318.pdf.

Consejería de Educación de la Junta de Andalucía (2011). Decreto 260/2011, de 26 de julio, por el que se establecen las enseñanzas artísticas superiores de Grado en Música en Andalucía. Boletín Oficial de la Junta de Andalucía, 165, 37-92. Disponible en http://www.juntadeandalucia.es/ boja/2011/165/d8.pdf.
García López, R. y Sales, A. (2009). La formación ética profesional desde la perspectiva del profesorado universitario. Teoría de la Educación, 21, 199-221.

Ministerio de Educación (2009). Real Decreto $1614 / 2009$, de 26 de octubre, por el que se establece la ordenación de las enseñanzas artísticas superiores reguladas por la Ley Orgánica 2/2006, de 3 de mayo, de Educación. Boletín Oficial del Estado, 259, 89.743-89.752. Disponible en http: / / www.boe.es/boe/dias/2009/10/27/pdfs/BOEA-2009-17005.pdf.

Ministerio de Educación (2010). Real Decreto $861 / 2010$, de 2 de julio, por el que se modifica el Real Decreto 1393/2007, de 29 de octubre, por el que se establece la ordenación de las enseñanzas universitarias oficiales. Boletín Oficial del Estado, 161, 58.454-58.468. Disponible en http:// www. boe.es/boe/dias / $2010 / 07 / 03 / \mathrm{pdfs} / \mathrm{BOE}$ A-2010-10542.pdf.

Ministerio de Educación (2010). Real Decreto $631 / 2010$, de 14 de mayo, por el que se regula el contenido básico de las enseñanzas artísticas superiores de Grado en Música establecidas en la Ley Orgánica 2/2006, de 3 de mayo, de Educación. Boletín Oficial del Estado, 137, 48.48048.500. Disponible en http://www.boe.es/boe/dias/ 2010/06/05/pdfs/BOE-A-2010-8955.pdf.

Ministerio de Educación, Gultura y Deporte (2014). Real Decreto 96/2014, de 14 de febrero, por el que se modifican los Reales Decretos 1027/2011, de 15 de julio, por el que se establece el Marco Español de Cualificaciones para la Educación Superior (MECES), y 1393/2007, de 29 de octubre, por el que se establece la ordenación de las enseñanzas universitarias oficiales. Boletín Oficial del Estado, 55, 20.151-20.154. Disponible en http://www.boe.es/boe/dias/ 2014/03/05/pdfs/BOE-A-2014-2359.pdf.

Ministerio de Educación, Cultura y Deporte (2015). Real Decreto 21/2015, de 23 de enero, por el que se modifica el Real Decreto 1614/2009, de 26 de octubre, por el que se establece la ordenación de las enseñanzas artísticas superiores reguladas por la Ley Orgánica 2/2006, de 3 de mayo, de Educación. Boletín Oficial del Estado, 33, 10.319-10.324. Disponible en http://www.boe.es/boe/dias/2015/02/07/ pdfs/BOE-A-2015-1157.pdf.

Ministerio de Educación y Ciencia (2007). Real Decreto 1393/2007, de 29 de octubre, por el que se establece la ordenación de las enseñanzas universitarias oficiales. Boletín Oficial del Estado, 260, 44.037-44.048. Disponible en http:// www.boe.es/boe/dias/2007/ $10 / 30 / \mathrm{pdfs} /$ A44037-44048.pdf.

Román, B. y Bara, F. E. (2012). Aporías de la formación ética en la universidad de hoy. Revista del Congrés Internacional de Docència Universitària $i$ Innovació (CIDUI), 1(1). Disponible en http://www.cidui.org/revista-cidui 12/index.php/cidui/ article/view/395.

Zaldívar, Á. (2005). Las enseñanzas musicales y el nuevo Espacio Europeo de Educación Superior: el reto de un marco organizativo adecuado y la necesidad de la investigación creativa y performativa. Revista Interuniversitaria de Formación del Profesorado, 52, 95-122. Disponible en http:// dialnet.unirioja.es/servlet/articulo? codigo $=1343170$.

Zaldívar, Á. (2006). El reto de la investigación creativa y performativa. Eufonía, 38, 87-94. 
Zaldívar, Á. (2008a). Adquirir, aplicar y producir conocimientos artísticos: propuestas de mejora a partir de las competencias de los distintos ciclos de los estudios superiores musicales conforme al proceso de Bolonia. En Actas del I Congreso de Educación e Investigación Musical. Madrid: IEMSEM/EE-UAM.

Zaldívar, Á. $\left(2008^{\mathrm{b}}\right)$. Investigar desde el arte. Anales (Real Academia Canaria de Bellas Artes de San Miguel Arcángel), 1(1), 57-64.

Zaldívar, Á. (2010). Investigar desde la práctica artística. En Libro de Actas. I Congreso Internacional Investigación en Música. Valencia: ISEACV.

Zaldívar, Á. (2011). Epílogo: Apuntes para un heptálogo del investigador. En V. Calvo y F. Labrador (Ed.). In_des_ar. Investigar desde el Arte. Madrid: Dykinson.

Zaldívar, Á. (2013). La práctica musical como tarea científica: investigando etnográficamente los procesos artísticos. En Actas del II Congreso de Educación e Investigación Musical CEIMUS. Madrid: Enclave Creativa. 


\section{Sobre el Autor}

\section{Álvaro Zaldívar Gracia}

Profesor Superior de Música por el Conservatorio Superior de Música de Barcelona y Doctor en Filosofía y Ciencias de la Educación por la Universidad de Valencia. Catedrático numerario de Historia de la Música y jefe del Departamento de Musicología e Investigación del Conservatorio Superior de Música de Murcia. Ha sido Inspector General de Conservatorios y Subdirector General de Personal Docente e Investigador del Ministerio de Educación y Ciencia, y Subdirector General de Enseñanzas Artísticas Superiores de la Comunidad de Madrid. Consejero de número de la Institución Fernando el Católico, es Académico numerario de la Real de Bellas Artes de Murcia, y Correspondiente de las Reales Academias de Bellas Artes de Zaragoza, Madrid y Extremadura. Miembro de Honor de la Asociación Española de Centros Superiores de Enseñanzas Artísticas, de la que fue cofundador y Vicepresidente, y Académico de Honor de la M.I. Academia de la Música Valenciana. Posee la Medalla de Oro del Real Conservatorio Superior de Música de Madrid y la Encomienda de la Orden Civil de Alfonso X el Sabio.

\section{Álvaro Zaldívar Gracia}

Conservatorio Superior de Música Manuel Massotti Littel

Alcalde Gaspar de la Peña, 9

30004 Murcia. España.

alvarozaldivar@hotmail.com

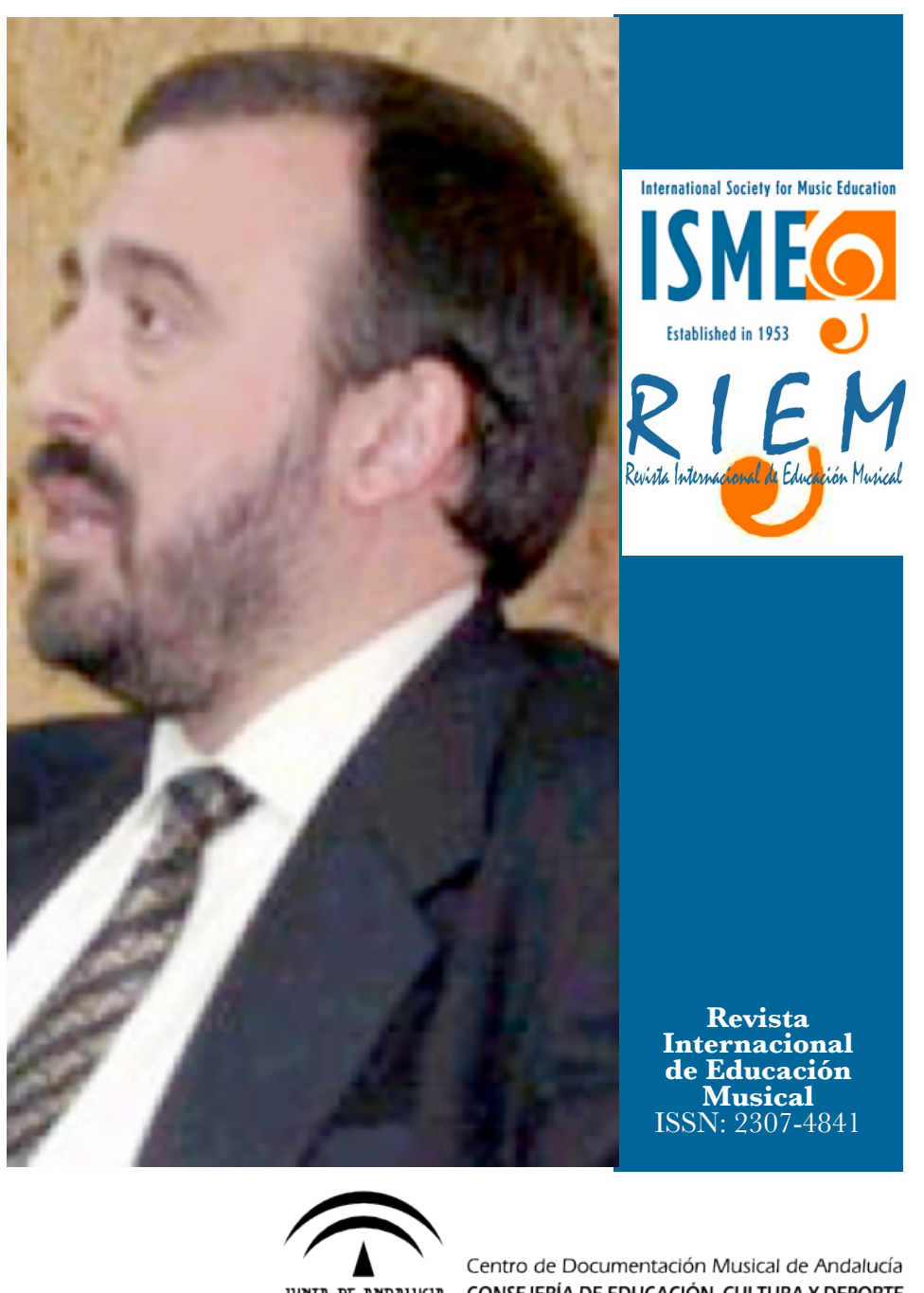

JUNTA DE AMDALUCIA CONSEJERIA DE EDUCACIÓN, CULTURA Y DEPORTE

\section{EQUIPO EDITORIAL}

Editor:

José Luis Aróstegui Plaza, Universidad de Granada (España)

\section{Editora Adjunta:}

Rosa María Serrano Pastor, Universidad de Zaragoza (España)

\section{Consejo Editorial}

Carlos Abril, Universidad de Miami (Estados Unidos)

María del Carmen Aguilar, Instituto Coral de Buenos Aires (Argentina)

Miquel Alsina Tarrés, Universidad de Gerona (España)

Carmen Carrillo Aguilera. Universidad Internacional de Cataluña (España)

Sergio Luiz Figueiredo, Universidad del Estado de Santa Catarina (Brasil)

Patricia Adelaida González, Universidad Autónoma de Chihuahua (México)

Claudia Gluschankof, Instituto Levinsky (Israel)

Josep Gustems Carnicer, Universidad de Barcelona (España)

María Cecilia Jorquera Jaramillo, Universidad de Sevilla (España)

Gotzon Ibarretxe Txakartegi, Universidad del País Vasco (España)

Ana Laucirica Larrinaga, Universidad Pública de Navarra (España)

Ana Lucia Louro, Universidad Federal de Santa María (Brasil)
Isabel Cecilia Martínez, Universidad Nacional de La Plata (Argentina)

Teresa Mateiro, Universidad del Estado de Santa Catarina (Brasil)

María Teresa Moreno, Universidad Laval (Canadá)

Graça Boal Palheiros, Instituto de Educación de Oporto (Portugal)

Jèssica Pérez Moreno, Universidad Autónoma de Barcelona (España) y Universidad de Londres (Reino Unido)

Gabriel Enrique Rusinek Milner, Universidad Complutense de Madrid (España)

Patrick K. Schmidt, Universidad de Ontario Occidental (Canadá)

Favio Shifres, Universidad Nacional de La Plata (Argentina)

Maria dels Àngels Subirats Bayego, Universidad de Barcelona (España)

António Ângelo Ferreira Vasconcelos, Instituto de Educación de Setúbal (Portugal)

Maria Helena Vieira, Universidad del Miño (Portugal)

Gloria Patricia Zapata Restrepo, Universidad de Antioquía (Colombia) 